\title{
Mulberry granules protect against diabetic cardiomyopathy through the AMPK/Nrf2 pathway
}

\author{
YANG LIU ${ }^{1}$, YAN-BO ZHAO ${ }^{2}$, SI-WANG WANG ${ }^{3}$, YU ZHOU $^{4}$, ZHI-SHU TANG $^{1}$ and FENG $\mathrm{LI}^{5}$ \\ ${ }^{1}$ School of Pharmacy, Shaanxi University of Chinese Medicine, Xianyang, Shaanxi 712000; ${ }^{2}$ Department of Emergency, \\ Renmin Hospital, Hubei University of Medicine, Shiyan, Hubei 442000; ${ }^{3}$ Department of Natural Medicine, School of Pharmacy, \\ The Fourth Military Medical University, Xi'an, Shaanxi 710032; ${ }^{4}$ Department of Otorhinolaryngology, \\ The People's Hospital of Ankang, Ankang, Shaanxi 725000; ${ }^{5}$ Department of Traditional Chinese Medicine, \\ Xijing Hospital, The Fourth Military Medical University, Xi'an, Shaanxi 710032, P.R. China
}

Received June 15, 2016; Accepted May 15, 2017

DOI: $10.3892 /$ ijmm.2017.3050

\begin{abstract}
Mulberry granules (MLD) is a traditional Chinese medicine prescription that has been used in the treatment of diabetes for many years. Recently, we found that MLD protected the heart from diabetes-associated cardiomyopathy when it was used to treat diabetes. However, the beneficial effects and possible mechanism remain unknown. To elucidate these effects, an experimental myocardial ischemia/reperfusion (MI/R) injury model in diabetes rats was used in this study. Male C57BL/6 mice were injected with streptozotocin to induce diabetes. The mice were pretreated with MLD for one month, and then exposed to $30 \mathrm{~min}$ of ischemia followed by $24 \mathrm{~h}$ of reperfusion. Infarct size, heart function and various cytokines in the heart were assessed. Expression of AMP-activated protein kinase (AMPK) and nuclear factor erythroid 2-related factor 2 (Nrf2) were investigated by western blotting. In vitro, MLD significantly cleared oxygen-free radicals in DPPH and luminol chemiluminescence models. In vivo, fasting blood glucose, fasting blood insulin and lipids were significantly decreased by MLD. The results showed that MLD improved the cardiac function and decreased myocardial infarct size in the diabetic mice subjected to MI/R. In addition, upon pretreatment with MLD before MI/R treatment, GSH, SOD, CAT and GR were significantly increased in a dose-dependent manner. Pretreatment with MLD also significantly induced the expression of Nrf2, and the cardioprotective effects of MLD were abolished in Nrf2-knockout mice. Furthermore,
\end{abstract}

Correspondence to: Professor Zhi-Shu Tang, School of Pharmacy, Shaanxi University of Chinese Medicine, Xianyang, Shaanxi 712000, P.R. China

Email: tzs6565@163.com

Professor Feng Li, Department of Traditional Chinese Medicine, Xijing Hospital, The Fourth Military Medical University, Xi'an, Shaanxi 710032, P.R. China

Email: feng_li3@aol.com

Key words: mulberry granules, diabetic cardiomyopathy, oxidative stress, AMP-activated protein kinase, nuclear factor erythroid 2-related factor 2 we also found that AMPK increase is upstream and was required for Nrf2 activation mediated by MLD. In conclusion, MLD protects against diabetic-associated cardiomyopathy by suppressing oxidative stress induced by hyperglycemia and MI/R through the AMPK/Nrf2 signaling pathway.

\section{Introduction}

In recent decades, the incidence of diabetes mellitus has increased rapidly, and by 2025 , the number of individuals with diabetes is estimated to be $330-380$ million. Diabetes is recognized as a potent and prevalent risk factor for ischemic heart disease. Among the 3.8 million deaths each year, $2 / 3$ are attributable to heart disease associated with diabetes (http://www.eatlas.idf.org/). Diabetic cardiomyopathy (DCM), associated with both type 1 and 2 diabetes, represents one of the major cardiovascular complications in diabetic patients and is characterized by both early-onset diastolic and lateonset systolic dysfunctions that may lead to heart failure $(1,2)$. Several mechanisms have been implicated in the pathogenesis of DCM. Among them, oxidative stress is involved in the etiology of diabetes-induced downregulation of heart function (3). The beneficial effects of several molecules on DCM have been attributed to the alleviation of oxidative stress (4).

Oxidative stress is defined as a serious imbalance between the production of endogenous reactive oxygen species (ROS) and antioxidants, and in favor of the former (5). Hyperglycemia, a major etiological component in the development of DCM, is known to promote the production of reactive nitrogen species (RNS) and ROS and/or to deplete antioxidant systems in many cell types. Overproduction of ROS is a causative factor in the development of DCM (6-8). Blocking ROS formation has been shown to prevent hyperglycemic damage (7). Even though antioxidant prevention or therapy has been explored for many years, there are few exogenous antioxidants that efficiently prevent DCM in the clinic to date. Accordingly, activation of the endogenous antioxidant pathways in the heart and the underlying molecular mechanisms have been proposed as an attractive strategy (9).

Morus alba L., a plant of the Moraceous family, has more than 4,000 years of history in China. Mulberry is cultivated for 
fruit production, and its leaf is traditionally used as the food of silkworms (10). Among the various nutritional components of mulberry, flavonoids are known to have effects as antioxidants or free radical scavengers (11). It also contains many phenolic antioxidants that can reduce cardiovascular disease. Several recent studies have shown that mulberry has antioxidant, anti-hyperglycemic, anti-inflammatory, neuroprotective, and hypolipidemic and non-toxic activities (12-14). Dietary mulberry has also been reported to have hypoglycemic, hypolipidemic and antioxidant effects (15). Based on LDL oxidation assay, mulberry showed relatively high antioxidant activity in comparison with 52 types of edible plant products in Japan (16). Because of these findings, the food of mulberry was developed into a type of granules, named mulberry granules (MLD), which is convenient to eat. MLD controls blood glucose levels in diabetes patients, and we also found their beneficial effects on DCM in our preliminary experiments. However, the beneficial effects and possible mechanism remain largely unknown. This study was carried out to explore the potential of MLD in the management of experimental myocardial ischemia/reperfusion (MI/R) injury in diabetes mice. This investigation may promote an understanding of the mechanisms underlying the protective effects of MLD against diabetes-associated cardiomyopathy.

\section{Materials and methods}

Chemicals and herbal materials. The fruits of the black mulberry (Morus nigra) were purchased from Xi'an Zaolutang Pharmaceutical Co, Ltd. (Xi'an, China) and were identified botanically by Professor Haifeng Tang (Department of Natural Medicine, School of Pharmacy, The Fourth Military Medical University, Xi'an, China). A voucher specimen (no. 1509252) was deposited at the Department of Natural Medicine, School of Pharmacy, The Fourth Military Medical University.

MLD was obtained from Shaanxi Junbisha Pharmaceutical Co., Ltd. (Xianyang, China). Chlorogenic acid (with a purity $>98 \%$ by HPLC analysis) was purchased from National Institute for the Control of Pharmaceutical and Biological Products (Beijing, China). HPLC-grade methanol and acetonitrile were obtained both from Thermo Fisher Scientific, Inc. (Waltham, MA, USA). Deionized water was purified using the Milli-Q Reagent Water system (Millipore, Bedford, MA, USA). Streptozotocin (STZ) was obtained from SigmaAldrich (St. Louis, MO, USA). Polyclonal rabbit anti-mouse AMP-activated protein kinase (AMPK; 2532), p-AMPK (2535), nuclear factor erythroid 2-related factor 2 (Nrf2; 12721) and $\beta$-actin (4970) antibodies were purchased from Cell Signaling Technology, Inc. (Danvers, MA, USA). Lactate dehydrogenase (LDH), creatinine kinase (CK)-MB, SOD, GSH, CAT and GR detection kit were purchased from Nanjing Jiancheng Bioengineering Institute (Nanjing, China).

HPLC analysis. For phytochemical analysis, MLD $(\sim 0.50 \mathrm{~g})$ was extracted with $50 \mathrm{ml}$ of $75 \%(\mathrm{w} / \mathrm{v})$ ethanol in a water bath at $80^{\circ} \mathrm{C}$ for $30 \mathrm{~min}$ with one shaking every $15 \mathrm{~min}$. After adjusting to ambient temperature, ethanol was then added to compensate for the lost weight during the extraction. Finally, the supernatant was filtered through a $0.22-\mathrm{mm}$ membrane filter and injected into the HPLC system for analysis.
HPLC analysis was performed on a Shimadzu LC-20AD system (Shimadzu, Kyoto, Japan). The analytes were separated on a Kromasil C18 (250 mm x $4.6 \mathrm{~mm}, 5 \mu \mathrm{m})$. The mobile phase consisted of A (acetonitrile) and B (water containing $0.3 \%$ phosphoric acid). The flow rate was $0.8 \mathrm{ml} / \mathrm{min}$ at $30^{\circ} \mathrm{C}$. $\mathrm{UV}$ absorbance was monitored at $254 \mathrm{~nm}$ for finger printing analysis and quantitative analysis. Chromatographic data were collected and processed by an Empower ${ }^{\mathrm{TM}}$ chromatographic working station (Waters, Milford, MA, USA).

Determination of 1,1-diphenyl-2-picryl-hydrazyl (DPPH) radical scavenging activity. The free radical scavenging activity of MLD was measured by DPPH assay as we previously described (17). DPPH-free radical agent was prepared as $0.0158 \mathrm{~g}$ dissolved in $50 \mathrm{ml} 95 \%$ (v:v) ethanol, and mixed with MLD solution with a ratio of 8:1. The mixture was shaken and kept in the dark at $37^{\circ} \mathrm{C}$ for $30 \mathrm{~min}$. Vitamin $\mathrm{C}(\mathrm{Vc})$ was employed as a control. The quantity of DPPH remaining in the mixed solution was measured at $517 \mathrm{~nm}$.

Superoxide anion radical-scavenging activity. Xanthine oxidase and xanthine-induced luminol chemiluminescence measurements were carried out as previously described (18) with some modifications. The concentration gradient of the sample and Vc were 9.6, 19.2, 28.8, 38.4 and $48 \mu \mathrm{g} / \mathrm{ml}$. The mixture, sample or $\mathrm{Vc}$ and $4.5 \mathrm{ml}$ of $50 \mathrm{mM}$ Tris- $\mathrm{HCl}$ buffer ( $\mathrm{pH} 8.2$ ) were mixed in a $10 \mathrm{ml}$ centrifuge tube, and then shaken and incubated at $25^{\circ} \mathrm{C}$ for $20 \mathrm{~min}$. Preheated $\left(25^{\circ} \mathrm{C}\right)$ $0.3 \mathrm{ml}$ of $3 \mathrm{mM}$ pyrogallol solution was then added. After standing for $5 \mathrm{~min}, 0.2 \mathrm{ml}$ of $10 \mathrm{M}$ of $\mathrm{HCl}$ was added to stop the reaction. The absorbance was measured at $325 \mathrm{~nm}$. As a control, $0.3 \mathrm{ml}$ of $10 \mathrm{mM} \mathrm{HCl}$ replaced the pyrogallol solution.

Animals and type 2 diabetic mouse model. The mice were obtained from the Experimental Animal Center of the Fourth Military Medical University. The experimental protocol was approved by the Ethics Committee for Animal Experimentation and was performed according to the Guidelines for Animal Experimentation of the Fourth Military Medical University and the National Institute of Health Guide for the Care and Use of Laboratory Animals (NIH publications no. 80-23) revised in 1996. The animals were housed under a 12-h light/dark cycle and temperature was kept at $25^{\circ} \mathrm{C}$.

For the STZ diabetic model, 6- to 8-week-old male C57BL/6 mice, weighing 23-25 g were used. They were randomly grouped into diabetic (model) or non-diabetic (control) mice as they received, for 5 consecutive days, an intraperitoneal injection of STZ (Sigma-Aldrich) at a dose of $50 \mathrm{mg} / \mathrm{kg}$ or vehicle $(0.1 \mathrm{~mol} / 1$ citrate buffer, $\mathrm{pH} 4.5)$ alone. After 4 weeks, blood glucose levels were measured using Bayer BREEZE $^{\circledR} 2$ meter (Bayer HealthCare LLC, Mishawaka, IN, USA) by tail vein blood sampling. Mice with blood glucose levels $>11.1 \mathrm{mM}$ were used as the model group in the present study.

MLD was dissolved in physiological saline before use. Animals were randomly divided into five groups: sham, $\mathrm{I} / \mathrm{R}, \mathrm{I} / \mathrm{R}+\mathrm{MLD}$ (30 mg/kg), I/R + MLD (20 mg/kg) and $\mathrm{I} / \mathrm{R}+\mathrm{MLD}(10 \mathrm{mg} / \mathrm{kg})$ groups. MLD was administered through oral administration for one month. Then, the animals were exposed to $30 \mathrm{~min}$ ischemia followed by $24 \mathrm{~h}$ of reperfusion 
using a previously described technique (19). After these operations, infarct size and heart function were estimated.

Blood analyses. Glycemic parameters including fasting blood glucose (FBG) and fasting blood insulin (FBI) were measured by enzymatic end procedure and enzyme-linked immunosorbent assay (ELISA) according to the manufacturer's instructions respectively. Homeostasis model of insulin resistance (HOMA-IR) was calculated as described: HOMA-IR (mass units $)=$ fasting insulin $(\mathrm{mIU} / \mathrm{ml}) \times$ fasting glucose $(\mathrm{mg} / \mathrm{dl}) / 405$. High-density lipoprotein cholesterol (HDL-C) and low-density lipoprotein cholesterol (LDL-C), total cholesterol (TC) and triglyceride (TG) in serum were determined using of the Bayer 1650 blood chemistry analyzer (Bayer, Tarrytown, NY, USA).

Cardiac function measurement. After induction of anesthesia with $5 \%$ chloral hydrate by intraperitoneal injection, cardiac parameters were detected by a biofunction experiment system MP100-CE (Biopac Systems Inc., Santa Barbara, CA, USA). Heart rate (HR), left ventricular systolic pressure (LVSP), left ventricular maximum upstroke and descent velocity $\left(+\mathrm{dP} / \mathrm{dt}_{\max } /-\mathrm{dP}_{\mathrm{dt}}\right.$ max $)$ were measured by computer algorithms and an interactive videographics program (Po-Ne-Mah Physiology Platform P3 Plus). MI size was determined by the Evans blue/TTC double-staining method as previously described after completion of functional determination (20).

Detection of $\mathrm{LDH}$ and $C K-\mathrm{MB}$ levels. Blood samples were collected from the abdominal aorta after $24 \mathrm{~h}$ of reperfusion and centrifuged at 3,250 x g for $10 \mathrm{~min}$. LDH and CK-MB levels in plasma were measured to evaluate myocardial damage using commercially available assay kits (Nanjing Jiancheng Bioengineering Institute).

Detection of GSH, GSSG, CAT, SOD and MDA. GSH, GSSG, CAT, SOD and MDA levels in cardiac muscle tissues or myocardial cell were detected by the corresponding kit according to the manufacturer's instructions.

Western blotting. After reperfusion, $\sim 50 \mathrm{mg}$ of myocardial tissue was collected and stored at $-80^{\circ} \mathrm{C}$. Radioimmunoprecipitation assay (RIPA) lysis buffer was used to extract the whole protein, and the concentration was determined by the BCA protein kit according to the manufacturer's instructions, and the mean concentration values were computed. The assessment was carried out twice for each sample, and the values were averaged. Then the samples were kept at $-80^{\circ} \mathrm{C}$.

Equal amount of protein was separated by $10 \%$ Tris-glycine SDS-PAGE polyacrylamide gel and transferred to polyvinylidene fluoride membranes (Invitrogen Life Technologies, Carlsbad, CA, USA). After blocking for $1.5 \mathrm{~h}$ with a $5 \%$ solution of skim milk, the membranes were incubated with the primary antibodies at $4^{\circ} \mathrm{C}$ overnight, followed by the horseradish peroxidase (HRP)-linked anti-goat antibody for $60 \mathrm{~min}$ at $37^{\circ} \mathrm{C}$. After washing with Tris-buffered saline, the relative intensity of the protein signals was normalized to the corresponding $\beta$-actin intensity and was quantified by densitometric analysis with the use of Quantity One software (Bio-Rad Laboratories, Inc., Hercules, CA, USA).
Statistical analysis. The experiment was performed at least three times. Data are expressed as means \pm standard deviation (SD), and were analyzed with SPSS statistical software (SPSS, Inc., Chicago, IL, USA). Data were compared by one-way analysis of variance (ANOVA). $\mathrm{P}<0.05$ was considered statistically significant.

\section{Results}

HPLC method validation and sample analysis. To confirm the accuracy of the equipment, a sample solution was successive injected for 5 times, and then the correlation coefficients between the reference HPLC fingerprint and the sample HPLC fingerprints were calculated. The values obtained were 0.997 , indicating that the accuracy of our measurements was satisfactory. To determine the stability of the sample solutions during the measurement period, at different timepoints $(0,3,6,9$ and $12 \mathrm{~h})$, five injections of the same sample solution were analyzed and the correlation coefficients were calculated. The values obtained were $0.972(0 \mathrm{~h}), 0.969(3 \mathrm{~h})$, $0.971(6 \mathrm{~h}), 0.971(9 \mathrm{~h})$ and $0.972(12 \mathrm{~h})$, which confirmed that during the measurement periods the sample solutions were stable. We also investigated the repeatability of the analytical method with 5 solutions from the same sample, and the values obtained were 0.997 (0 h), 0.998 (3 h), 0.999 (6 h), 0.998 (9 h), and $0.999(12 \mathrm{~h})$, which confirmed that the method was repeatable. Using the method described above, the MLD sample was analyzed. The HPLC fingerprinting profiles are shown in Fig. 1. Chlorogenic acid was used for chemical standardization of the MLD preparation. The similarity between the values obtained for the 10 samples was higher than 0.998 . This indicate a high consistency and stability between the batches of MLD tested.

Antioxidant activities. To measure radical scavenging activities, several in vitro antioxidant assays were tested. DPPH is a stable free radical and is often used to evaluate the antioxidant activity of natural compounds. As shown in Fig. 2A, the activity of MLD in reducing the DPPH free radical was as effective as Vc. Superoxide anion radical and hydroxyl radical are two types of the most important free radicals in organisms. Luminol chemiluminescence models producing hydroxyl and superoxide anion radicals were also used to evaluate the free radical-scavenging activity of MLD. Fig. 2B shows a high degree of hydroxyl radical activity. Superoxide anion radical-scavenging activity of $\mathrm{Vc}$ was more efficient than that of MLD (Fig. 2C). The results showed that MLD had high antioxidant activity in vitro.

Effects of MLD on FBG, FBI and lipids. Several symptoms characteristic of hyperglycemia, hyperlipidemia, polydipsia and polyphagia were observed in the type 2 diabetic mice. There was no statistically significant difference in FBG, FBI and lipids among the group at the beginning of the experiment $(\mathrm{P}>0.05)$. The levels of FBG and FBI in the type 2 diabetic model mice were higher than those in the control mice at the end of 4 weeks $(\mathrm{P}<0.01)$. FBG and FBI were significantly lower in the MLD groups than levels in the diabetic mice at 4 weeks $(\mathrm{P}<0.01)$ (Fig. 3A and B). The HOMA-IR levels were also significantly decreased in the MLD groups, as compared 


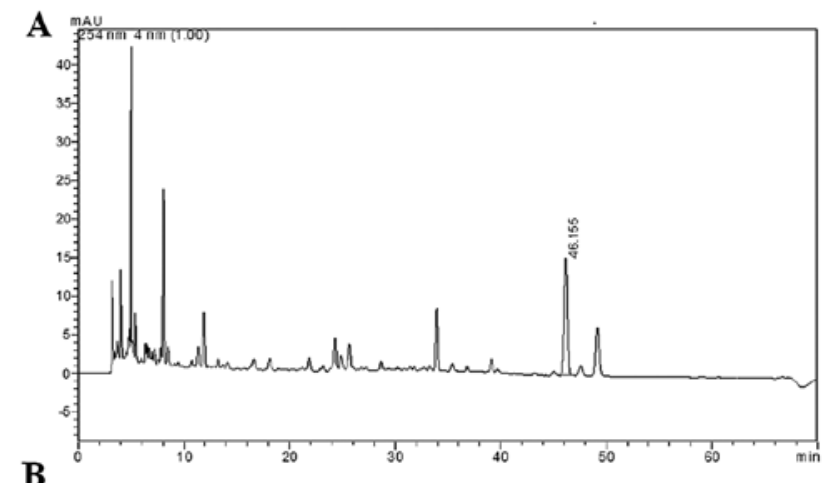

B

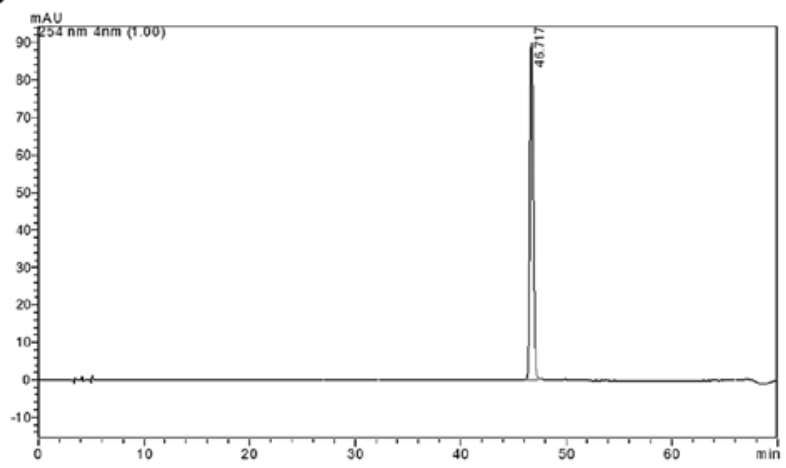

Figure 1. HPLC chromatogram of mulberry granules (MLD). (A) HPLC fingerprint analysis of the MLD. (B) HPLC fingerprint analysis of chlorogenic acid. Peaks were detected at $254 \mathrm{~nm}$.

with the diabetic model group (Fig. 3C; $\mathrm{P}<0.01$ ). These results indicated that administration of MLD in diabetic mice resulted in a significant improvement in the level of insulin resistance when compared to the diabetic group.

In the type 2 diabetic model group, TG, TC and LDL-C levels were significantly increased compared to these levels in the control group (Fig. 3D-F; P<0.01). There was no significant difference in HDL-C level between the control and diabetic model group (Fig. 3G). After a 4-week treatment with MLD, the TG, TC and LDL-C levels were significantly decreased compared to levels in the diabetic group $(\mathrm{P}<0.01$; 20 and $30 \mathrm{mg} / \mathrm{kg}$ MLD groups). After treatment with 20 and $30 \mathrm{mg} / \mathrm{kg}$ MLD, HDL-C was significantly increased $(\mathrm{P}<0.01)$. These results showed that type 2 diabetes was accompanied by insulin resistance and lipid metabolism disorder and that MLD improved the negative influences to some degree.

$M L D$ improves the cardiac function after MI/R in the diabetic mice. Cardiac function was examined to study whether MLD has cardioprotective effects against I/R injury in diabetic mice. As shown in Fig. 4, the $\mathrm{LVDP},+\mathrm{LV} \mathrm{dP/dt} \mathrm{max}_{\max }$ and $-\mathrm{LV} \mathrm{dP/dt} \mathrm{d}_{\max }$ were significantly decreased after $I / R$ in the diabetic mice $(\mathrm{P}<0.01)$. Compared to the sham group, $\mathrm{I} / \mathrm{R}$ injury caused a

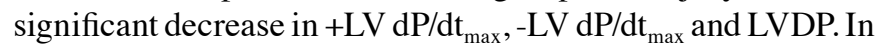
the MLD treatment groups these cardiac function parameters were improved significantly at the end of $3 \mathrm{~h}$ of reperfusion compared with the I/R group ( $\mathrm{P}<0.01 ; 20$ and $30 \mathrm{mg} / \mathrm{kg}$ MLD groups). These results showed that MLD improved cardiac functional recovery in the diabetic mice subjected to I/R.

$M L D$ inhibits myocardial injury following $M I / R$ in diabetic mice. As myocardial function is improved by MLD, the
A

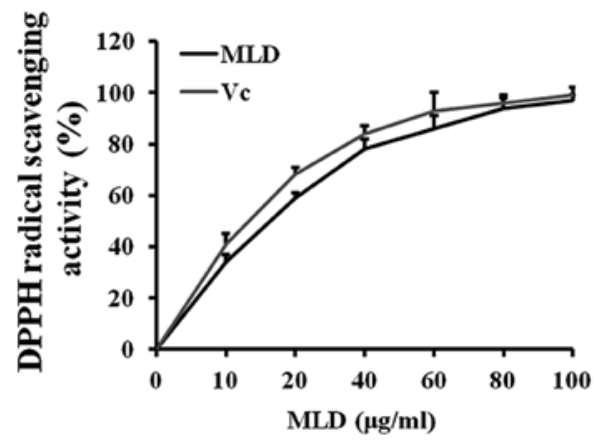

B

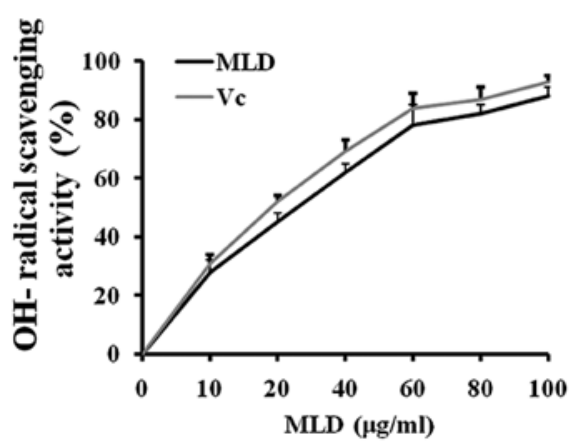

C

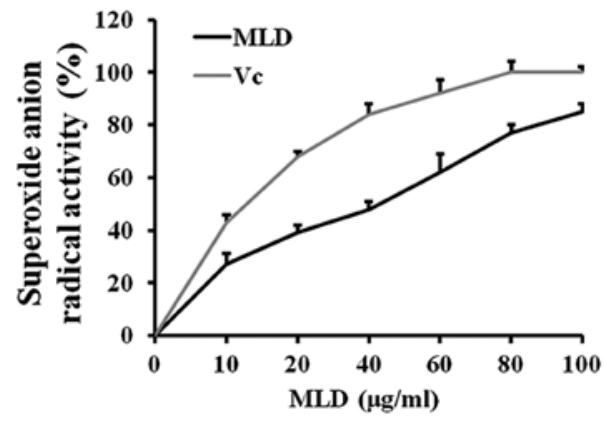

Figure 2. Antioxidant activities of mulberry granules (MLD) in vitro. (A) DPPH radical scavenging activity, (B) OH-radical scavenging activity and $(C)$ superoxide anion radical scavenging activity were determined. Data are the mean values of triplicate and expressed as mean $\pm \mathrm{SD}$.

infarct size was measured and is represented as a percentage of infarct area/area at risk. As shown in Fig. 5A, I/R caused significant myocardial infarction in the diabetic mice exposed to $\mathrm{I} / \mathrm{R}$ compared with the sham group $(\mathrm{P}<0.01)$. However, MLD significantly reduced the infarct size compared to the $\mathrm{I} / \mathrm{R}$ group $(\mathrm{P}<0.01 ; 20$ and $30 \mathrm{mg} / \mathrm{kg}$ MLD groups).

The activities of CK-MB and LDH contents in serum are commonly used to monitor damage of the myocardium. Relative to the sham group, CK-MB and LDH contents were significantly increased by $I / R$ in the diabetic mice $(\mathrm{P}<0.01)$ (Fig. 5B and $\mathrm{C})$. MLD significantly reversed these abnormal changes compared with the $I / R$ group $(\mathrm{P}<0.01 ; 20$ and $30 \mathrm{mg} / \mathrm{kg}$ MLD groups).

MDA, a marker of lipid peroxidation and free radical activity, was also assessed in this study. As shown in Fig. 5D, $\mathrm{I} / \mathrm{R}$ caused a significantly increase in MDA levels in the diabetic model mice $(\mathrm{P}<0.01)$, indicating that oxidative stress was induced by $\mathrm{I} / \mathrm{R}$ and diabetes. After pretreatment with MLD for 4 weeks, the MDA level was significantly decreased in a dose-dependent manner $(\mathrm{P}<0.01)$. 
A

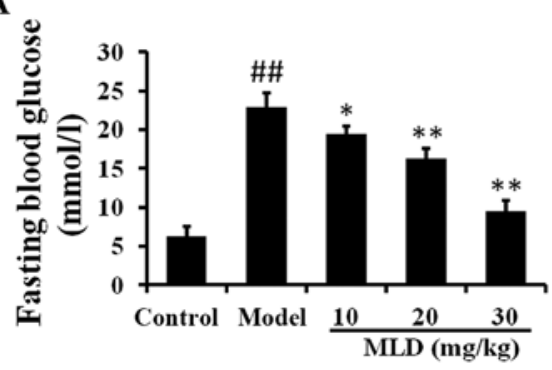

C

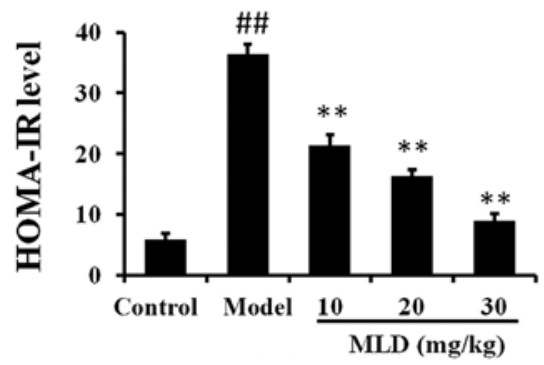

$\mathbf{E}$

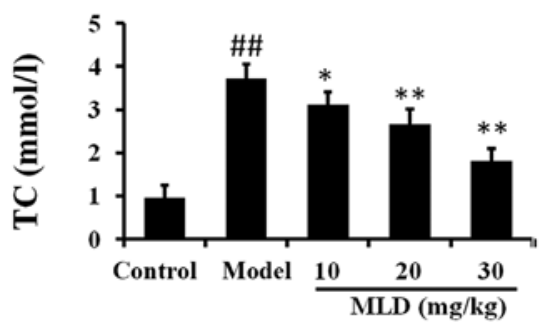

G

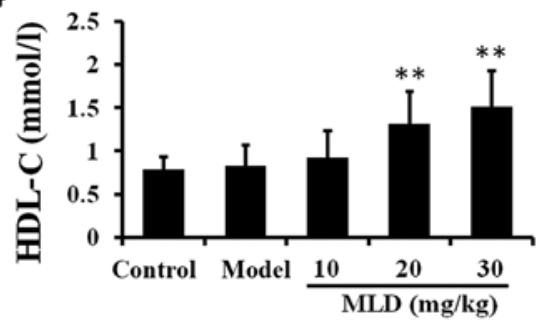

B

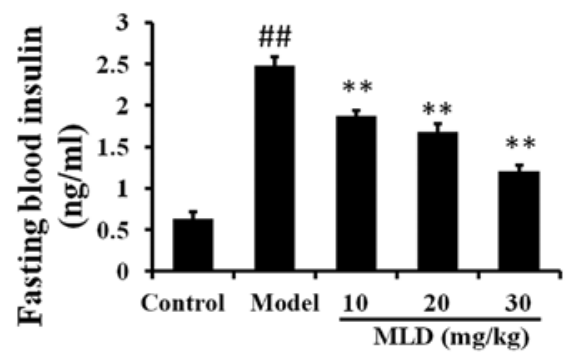

D

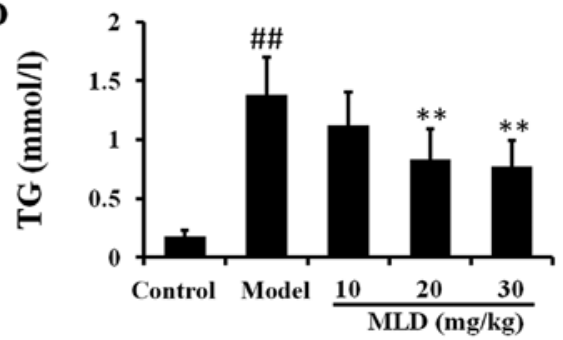

F

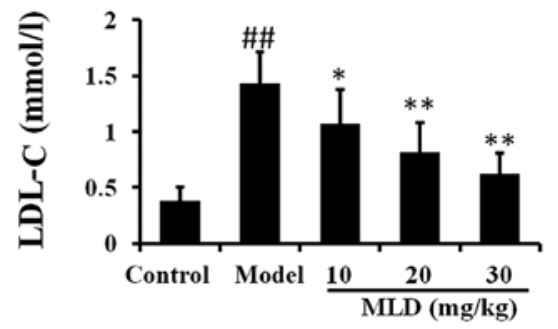

Figure 3. Effects of mulberry granules (MLD) on fasting blood glucose (FBG), fasting blood insulin (FBI) and lipids in diabetic mice. (A) FBG, (B) FBI, (C) homeostasis model of insulin resistance (HOMA-IR), (D) triglyceride (TG) levels in blood (mmol/l), (E) total cholesterol (TC) levels in blood (mmol/l), (F) low-density lipoprotein cholesterol (LDL-C) levels in blood (mmol/l) and (G) high-density lipoprotein cholesterol (HDL-C) levels in blood (mmol/l). There were 6 mice in every group. Data are represented as mean $\pm \mathrm{SD}$. ${ }^{\# \#} \mathrm{P}<0.01$ vs. control group; ${ }^{*} \mathrm{P}<0.05,{ }^{* *} \mathrm{P}<0.01$ vs. the model group.

A

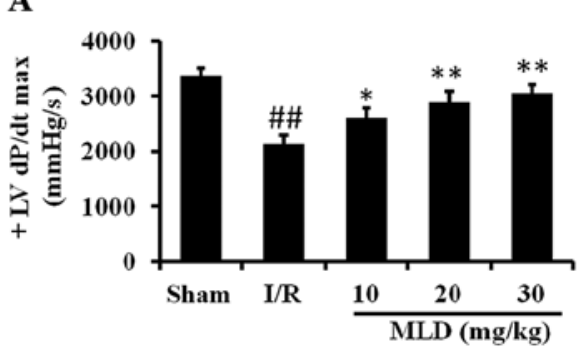

B

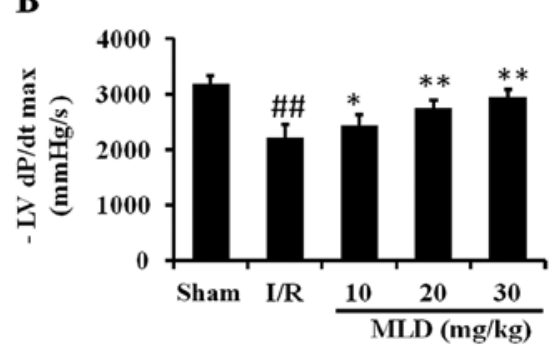

C

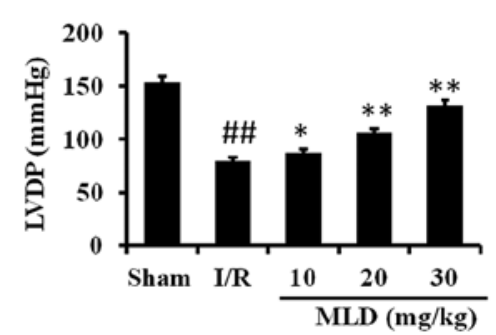

Figure 4. Effects of mulberry granules (MLD) on left ventricular maximum upstroke and descent velocity (+LV dP/dt $\left.t_{\max },-L V \mathrm{dP} / \mathrm{dt}_{\max }\right)$ and left ventricular systolic pressure (LVDP) in diabetic mice subjected to ischemia/reperfusion (I/R). (A) $+\mathrm{LV} \mathrm{dP/dt} t_{\max },(\mathrm{B})-\mathrm{LV} \mathrm{dP} / \mathrm{dt}_{\max }$ and (C) LVDP. There were 6 mice in every group. Data are represented as mean $\pm \mathrm{SD}$. ${ }^{\# \prime} \mathrm{P}<0.01$ vs. control group; ${ }^{*} \mathrm{P}<0.05,{ }^{* *} \mathrm{P}<0.01$ vs. the model group.

MLD enhances the antioxidant capacity in cardiac tissues. To test whether the protective effects of MLD against I/R-induced heart injury are due to its antioxidative activity, several antioxidant proteins were assessed. As shown in Fig. 6, the activities of GSH, SOD, CAT and GR were significantly decreased by $\mathrm{I} / \mathrm{R}$ in the diabetes mice $(\mathrm{P}<0.01)$. Pretreatment with MLD before I/R reversed these effects, which significantly included upregulation of actions of these enzymes $(\mathrm{P}<0.01 ; 20$ 
A

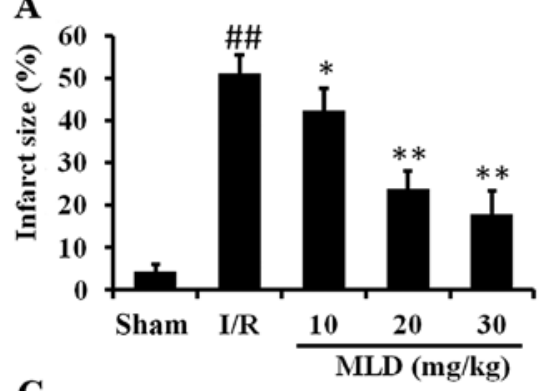

C

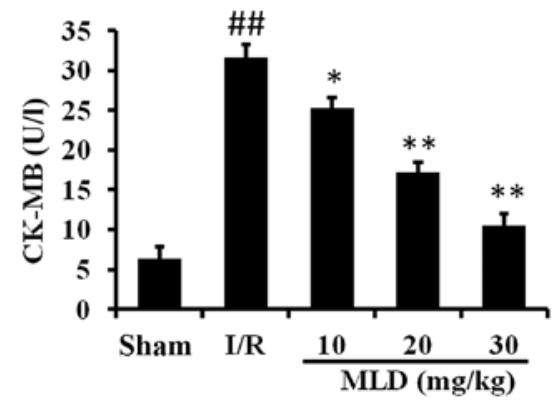

B
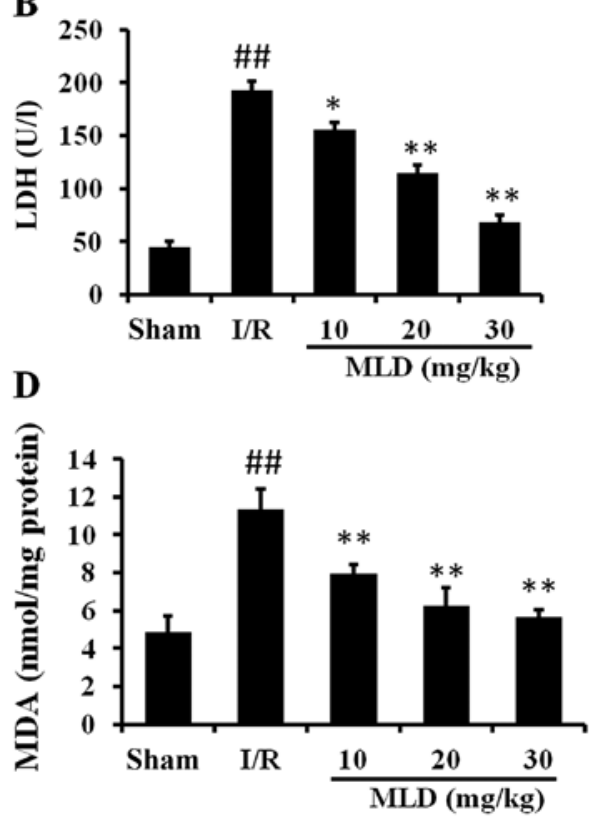

Figure 5. Effects of mulberry granules (MLD) on myocardial ischemia/reperfusion (MI/R) injury in diabetic mice. Different doses of MLD were administered orally for a 4-week duration of treatment after induction of diabetes. (A) Infarct size as stained by Evans blue and TTC, (B) lactate dehydrogenase (LDH) level (C) creatinine kinase (CK)-MB level and (D) MDA level in diabetic mice subjected to I/R without or with pretreatment of MLD. There were 6 mice in every group. Data are represented as mean $\pm \mathrm{SD}$. ${ }^{\# \#} \mathrm{P}<0.01$ vs. control group; ${ }^{*} \mathrm{P}<0.05,{ }^{* *} \mathrm{P}<0.01$ vs. model group.

A

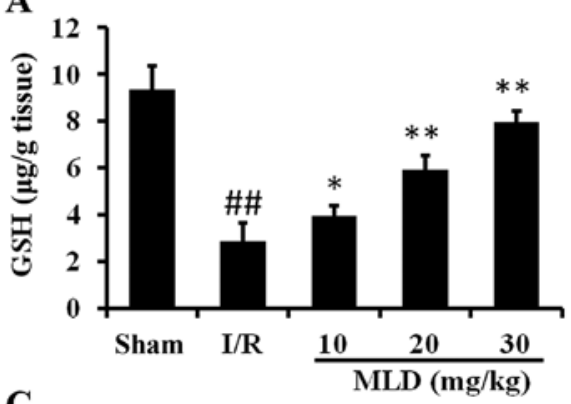

C

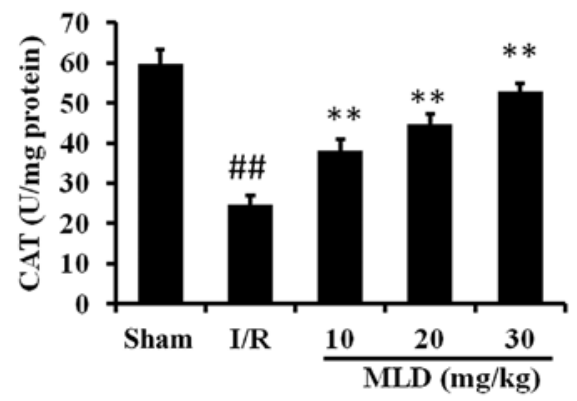

B
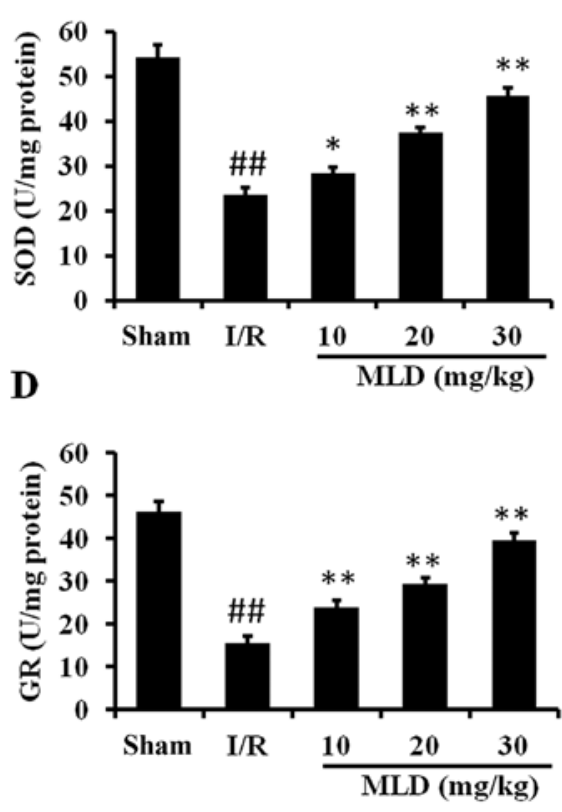

Figure 6. Effects of mulberry granules (MLD) on antioxidant protein expression in diabetic mice. (A) GSH expression, (B) SOD expression, (C) CAT expression and (D) GR expression in diabetic mice subjected to I/R without or with pretreatment of MLD. There were 6 mice in every group. Data are represented as mean $\pm \mathrm{SD}$. ${ }^{\# /} \mathrm{P}<0.01$ vs. sham group; ${ }^{*} \mathrm{P}<0.05,{ }^{* *} \mathrm{P}<0.01$ vs. ischemia/reperfusion (I/R) group.

and $30 \mathrm{mg} / \mathrm{kg}$ MLD groups). These findings suggest that MLD considerably improves cellular antioxidative defense capacity against oxidative stress.

$M L D$ activates the Nrf2 pathway. Nrf2 is a major regulator transcription factor against oxidative stress and the transcription factor Nrf2 plays a pivotal role in the induction of several antioxidant enzymes, including GSH, SOD, and CAT.
Therefore, we examined the effect of MLD on Nrf2 expression to assess the mechanism responsible for the antioxidative effect of MLD. As the results show, I/R caused a slight increase in Nrf2 expression in the diabetic model mice (Fig. 7A). Pretreatment with MLD before I/R significantly increased expression of Nrf2 $(\mathrm{P}<0.01)$. Subsequently, we compared the severity of heart and antioxidant protein levels in the WT and Nrf2-knockout (KO) mice to determine the role of Nrf2 in 

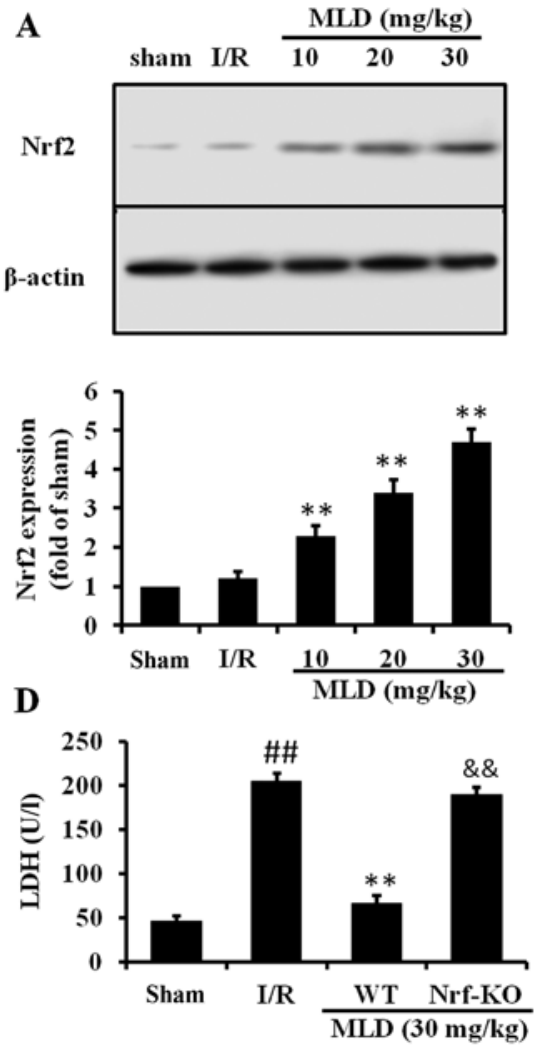
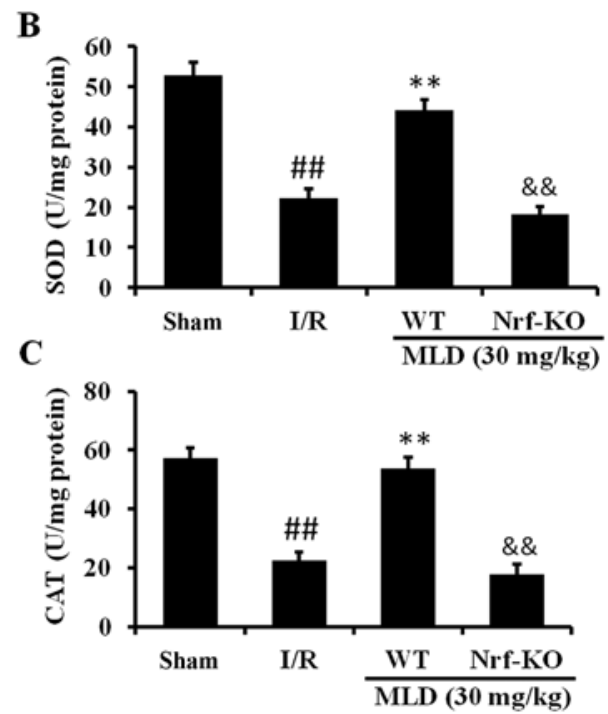

Figure 7. Effects of mulberry granules (MLD) on nuclear factor erythroid 2-related factor 2 (Nrf2) pathway. After different treatments, the heart was collected and homogenized. (A) The effects of different doses of MLD on the expression of Nrf2 in the heart subjected to ischemia/reperfusion (I/R). WT diabetic mice and Nrf2-knockout (KO) diabetic mice were pretreated with MLD (30 mg/kg), and then subjected to I/R. (B) SOD, (C) CAT and (D) lactate dehydrogenase ( $\mathrm{LDH})$ levels were measured in the different gouprs. There were 6 mice in every group. Data are represented as mean $\pm \mathrm{SD}$. ${ }^{\# \#} \mathrm{P}<0.01 \mathrm{vs}$. sham group; ${ }^{* *} \mathrm{P}<0.01$ vs. I/R group; ${ }^{\& \&} \mathrm{P}<0.01$ vs. WT group.

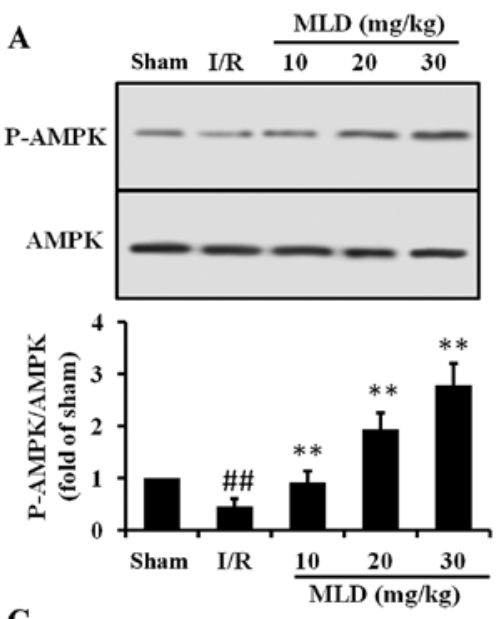

C

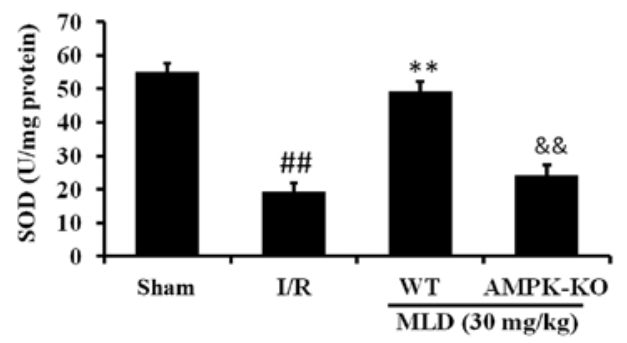

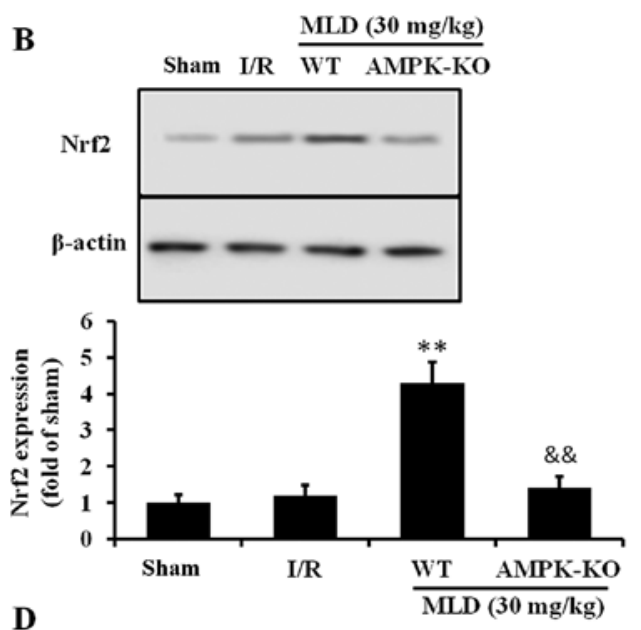

D

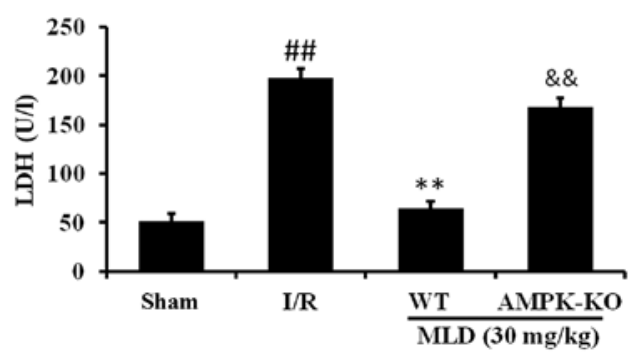

Figure 8. Mulberry granules (MLD) activate the nuclear factor erythroid 2-related factor 2 (Nrf2) pathway through AMP-activated protein kinase (AMPK), (A) Effects of different doses of MLD on the expression of Nrf2 in the heart subjected to ischemia/reperfusion (I/R). WT diabetic mice and Nrf2-knockout (KO) diabetic mice were pretreated with MLD $(30 \mathrm{mg} / \mathrm{kg}$ ), and then subjected to I/R. (B) Expression of Nrf2 in WT diabetic mice and Nrf2-KO diabetic mice. (C) SOD levels in WT diabetic mice and Nrf2-KO diabetic mice. (D) Lactate dehydrogenase (LDH) levels in WT diabetic mice and Nrf2-KO diabetic mice. There were 6 mice in every group. Data are represented as mean $\pm \mathrm{SD} .{ }^{\# \#} \mathrm{P}<0.01$ vs. sham group; ${ }^{* *} \mathrm{P}<0.01 \mathrm{vs}$. I/R group; ${ }^{\text {\&\&}} \mathrm{P}<0.01$ vs. WT group. 
MLD. The results of the ELISA assay revealed an increase in the levels of SOD and CAT in the WT diabetic mice subjected to I/R. However, the levels of these two cytokines were significantly lower in the Nrf2-KO mice compared with the WT mice (Fig. 7B and C). Moreover, the LDH level in the Nrf2-KO mice was significantly higher than that in the WT mice (Fig. 7D). These results demonstrated that the cardioprotective effects of MLD may be through the Nrf2 pathway.

MLD activates the Nrf2 pathway through AMPK. To investigate whether AMPK may be responsible for the protective effect of MLD, protein kinase activities in cardiac tissues were assessed by determining the phosphorylated form of AMPK. As shown in Fig. 8A, MLD administered p.o. for 4 weeks significantly $(\mathrm{P}<0.01)$ reversed the decreased phosphorylation of AMPK caused by I/R. To determine whether activation of Nrf2 is due to increased AMPK mediated by MLD, AMPK-KO mice were used. As the results show, AMPK-KO abolished the Nrf2 expression and cardioprotective effects (Fig. 8B and C) induced by AMPK, suggesting that the AMPK increase is upstream and is required for Nrf2 activation mediated by MLD.

\section{Discussion}

Diabetic patients have a 2- to 5-fold increased risk of developing cardiovascular diseases compared with non-diabetic patients, which include hypertensive heart disease, atherosclerosis and DCM (20). DCM is particularly difficult to manage due to its asymptomatic nature and its ability to progress into HR $(21,22)$. Several cardiovascular pathological consequences of diabetes such as hypertension affect the heart to varying degrees. However, hyperglycemia, as an independent risk factor, directly causes cardiac damage and leads to DCM (6). Hyperglycemia induces overproduction of reactive oxygen and nitrogen species, and then an outbreak of oxidative stress in the heart. Oxidative stress causes abnormal expression of genes or proteins, altered signal transduction and programmed myocardial cell death (23). Thus, myocardial cell loss plays a critical role in the onset and development of DCM. For the treatment of DCM, advances in the application of drugs against hyperglycemia-induced oxidative myocardial injury may be useful.

The edible product of mulberry, which contains flavonoids and phenols, is delicious and popular in many countries. It has been used to treat diabetes mellitus in ancient prescriptions and folk medicine. However, the food from mulberry physically deteriorates quickly following maturation, and does not favor preservation. Thus, mulberry was developed into a type of granules, MLD, which can be preserved for a long time. We found that it could protect diabetics from cardiovascular disease. But the cardioprotective effects and possible mechanism are largely unknown. In this study, HPLC was used for fingerprinting analysis and MI/R injury in a diabetic mouse model was used to evaluate its efficacy and possible mechanism.

There are several mechanisms involved in the function of antioxidants, one of which is the scavenging of ROS and free radicals. To evaluate the activity of MLD in scavenging free radical, two free radical-generating models were used.
DPPH, a stable free radical, is commonly used in testing free radical-scavenging activity. Any molecule that can transfer electrons or hydrogen atoms to DPPH may react with it and reduce the absorbance at $517 \mathrm{~nm}$ (24). When MLD was tested for DPPH-scavenging ability, it showed a strong DPPH-scavenging ability, which was similar to that of Vc. In this study, we also used xanthine oxidase-induced luminol chemiluminescence model. Compared with Vc, MLD showed a certain degree of free radical-scavenging activity. These results demonstrated that MLD could scavenge free radical directly in vitro.

To evaluate the cardioprotective effects of MLD, MI/R was induced in diabetic mouse. We found that MLD reduced FBG, FBI and lipids which were induced by STZ in the diabetic mice. We also found that MLD significantly improved cardiac dysfunction, including $+\mathrm{LV} \mathrm{dP/dt} t_{\max },-\mathrm{LV} \mathrm{dP/dt} t_{\max }$ and LVDP. Cytosolic enzymes, such as LDH and CK-MB, serve as diagnostic markers of myocardial ischemia injury. Upon injury, the cell membrane becomes permeable or ruptures, and then the enzymes leak out from the damaged myocardial tissues to the blood stream. Thus, they are considered as important indicators to determine reversible vs. irreversible damage to cells. In this study, LDH and CK-MB were increased by I/R in the diabetic mice, suggesting that $\mathrm{I} / \mathrm{R}$ and diabetes cause serious injury to the heart. However, MLD reversed those changes. To the best of our knowledge, this was the first study to demonstrate the protective role of MLD in diabetic mice with MI/R injury.

Chronic hyperglycemia has been reported to trigger oxidative stress either by direct generation of ROS or by altering the redox balance (25). The ability of antioxidants to inhibit hyperglycemia-derived injury has raised the possibility of new therapeutic treatments for diabetic heart diseases. In the present study, the ischemic heart of the diabetic mice showed a significant increase in the lipid peroxidation marker, MDA, with concomitant decreased GSH, SOD, CAT and GSR. MLD supplementation markedly attenuated I/R-induced oxidative stress by preventing GSH depletion and enhancement of the enzymatic antioxidants in the diabetic mice. These results together with the in vitro results indicated that the cardioprotective effects of MLD may occur by increasing the expression of endogenous antioxidant enzymes and scavenging free radical in the body directly.

Nrf2, a key transcriptional regulatory protein, activates antioxidant enzyme genes in response to oxidative stress (26). It is broadly expressed in multiple tissues but is only activated in response to various electrophilic agents and oxidative stress including ROS, some antioxidants and certain disease processes. Upon activation, Nrf2 through its interaction with the antioxidant response element (ARE), mediates the induction of a series of cytoprotective proteins including phase II enzymes, such as SOD, GSH and CAT $(27,28)$. These studies suggested that activation of the Nrf2/ARE pathway may be involved in the protein expression of SOD, CAT, GSH and GSR induced by MLD treatment. In the present study, the results showed that MLD upregulated Nrf2 expression in the hearts of the diabetic mice subjected to $\mathrm{I} / \mathrm{R}$, and also showed that expression of antioxidant proteins in Nrf2-KO mice was lower than that in WT mice. Under the condition of the same treatment, the level of injury in Nrf2-KO mice was much more serious than that in the WT mice. These results demonstrated 
that the cardioprotective effects of MLD may be through the activation of Nrf2 and its downstream proteins.

AMPK, a key factor in fat and carbohydrate metabolism that acts as a master switch of cellular metabolism, plays an important role in mediating the change between anabolic and catabolic states. Several factors activate the kinase, mainly cellular energy stress during physiological (exercise) or pathological (ischemia) situations, leading to a rise in AMP/ATP and ADP/ATP ratios (29). Once activated, AMPK induces several cellular signaling cascades and then activates metabolic key enzymes, and gene transcription, thus protecting the cell from I/R injury or other injury factors. A previous study also showed that defects in AMPK signaling result in the metabolic abnormalities of type 2 diabetes (30). In the present study, we demonstrated that MLD induced the phosphorylation of AMPK. To further clarify the role of AMPK in activating Nrf2, AMPK-KO mice were used. We found that the effect of MLD on expression of Nrf2 was abolished by AMPK-KO and also the cardioprotective effects.

In conclusion, a simple and rapid HPLC method was developed and validated the active compounds in MLD. MLD improved insulin resistance, decreased blood glucose, TG, TC and LDL levels in a diabetic mouse model. MLD has a protective effect on DCM by inhibiting hyperglycemiainduced oxidative stress through activation of the AMPK/ Nrf2 pathway. Given the key role of oxidative stress in the progression of DCM, MLD can be potentially used as an effective drug for the treatment of type 2 diabetes mellitus and cardiovascular diseases.

\section{Acknowledgements}

This study was supported by the ' 13115 ' Technology Innovation Project of Engineering Research Center of Shaanxi Province (no. 2013ZDGC-01).

\section{References}

1. Pappachan JM,Varughese GI,Sriraman R and Arunagirinathan G: Diabetic cardiomyopathy: pathophysiology, diagnostic evaluation and management. World J Diabetes 4: 177-189, 2013.

2. Galderisi M: Diastolic dysfunction and diabetic cardiomyopathy: evaluation by Doppler echocardiography. J Am Coll Cardiol 48: 1548-1551, 2006.

3. Ayaz M, Ozdemir S, Ugur M, Vassort G and Turan B: Effects of selenium on altered mechanical and electrical cardiac activities of diabetic rat. Arch Biochem Biophys 426: 83-90, 2004.

4. Ceriello A: New insights on oxidative stress and diabetic complications may lead to a 'causal' antioxidant therapy. Diabetes Care 26: 1589-1596, 2003.

5. Vassort G and Turan B: Protective role of antioxidants in diabetes-induced cardiac dysfunction. Cardiovasc Toxicol 10: 73-86, 2010

6. Cai L and Kang YJ: Oxidative stress and diabetic cardiomyopathy: a brief review. Cardiovasc Toxicol 1: 181-193, 2001

7. Nishikawa T, Edelstein D, Du XL, Yamagishi S, Matsumura T, Kaneda Y, Yorek MA, Beebe D, Oates PJ, Hammes HP, et al: Normalizing mitochondrial superoxide production blocks three pathways of hyperglycaemic damage. Nature 404: 787-790, 2000

8. Inoguchi T, Li P, Umeda F, Yu HY, Kakimoto M, Imamura M Aoki T, Etoh T, Hashimoto T, Naruse M, et al: High glucose level and free fatty acid stimulate reactive oxygen species production through protein kinase C-dependent activation of NAD $(\mathrm{P}) \mathrm{H}$ oxidase in cultured vascular cells. Diabetes 49: 1939-1945, 2000.

9. Cai L: Diabetic cardiomyopathy and its prevention by metallothionein: experimental evidence, possible mechanisms and clinical implications. Curr Med Chem 14: 2193-2203, 2007.
10. Arabshahi-D S, Vishalakshi Devi D and Urooj A: Evaluation of antioxidant activity of some plant extracts and their heat, $\mathrm{pH}$ and storage stability. Food Chem 100: 1100-1105, 2007.

11. Shibata Y, Kume N, Arai H, Hayashida K, Inui-Hayashida A, Minami M, Mukai E, Toyohara M,Harauma A,Murayama T, et al: Mulberry leaf aqueous fractions inhibit TNF-alpha-induced nuclear factor kappaB (NF-kappaB) activation and lectin-like oxidized LDL receptor-1 (LOX-1) expression in vascular endothelial cells. Atherosclerosis 193: 20-27, 2007.

12. Du J, He ZD, Jiang RW, Ye WC, Xu HX and But PP: Antiviral flavonoids from the root bark of Morus alba L. Phytochemistry 62: $1235-1238,2003$

13. Pan G and Lou C: Isolation of an 1-aminocyclopropane-1-carboxylate oxidase gene from mulberry (Morus alba L.) and analysis of the function of this gene in plant development and stresses response. J Plant Physiol 165: 1204-1213, 2008.

14. Sun F, Shen L and Ma Z: Screening for ligands of human aromatase from mulberry (Mori alba L.) leaf by using high-performance liquid chromatography/tandem mass spectrometry. Food Chem 126: 1337-1343, 2011.

15. El-Beshbishy HA, Singab AN, Sinkkonen J and Pihlaja K: Hypolipidemic and antioxidant effects of Morus alba L. (Egyptian mulberry) root bark fractions supplementation in cholesterol-fed rats. Life Sci 78: 2724-2733, 2006.

16. Enkhmaa B, Shiwaku K, Katsube T, Kitajima K, Anuurad E, Yamasaki M and Yamane Y: Mulberry (Morus alba L.) leaves and their major flavonol quercetin 3-(6-malonylglucoside) attenuate atherosclerotic lesion development in LDL receptor-deficient mice. J Nutr 135: 729-734, 2005.

17. Gulcin I: Antioxidant properties of resveratrol: a structure-activity insight. Innov Food Sci Emerg 11: 210-218, 2010.

18. Banu S, Greenway GM and Wheatley RA: Luminol chemiluminescence inducedby immobilised xanthine oxidase. Anal Chim Acta 541: 89-95, 2005.

19. Huang XL, Wang W and Zhou YW: Protective effect of epimedium flavonoids injection on experimental myocardial infarction rats. Zhongguo Zhong Xi Yi Jie He Za Zhi 26: 68-71, 2006 (In Chinese).

20. Trachanas K, Sideris S, Aggeli C, Poulidakis E, Gatzoulis K, Tousoulis D and Kallikazaros I: Diabetic cardiomyopathy: from pathophysiology to treatment. Hellenic J Cardiol 55: 411-421, 2014.

21. Aneja A, Tang WH, Bansilal S, Garcia MJ and Farkouh ME: Diabetic cardiomyopathy: insights into pathogenesis, diagnostic challenges, and therapeutic options. Am J Med 121: 748-757, 2008.

22. Arrington $\mathrm{CB}$, Dowse BR, Bleyl SB and Bowles NE: Non-synonymous variants in pre-B cell leukemia homeobox (PBX) genes are associated with congenital heart defects. Eur J Med Genet 55: 235-237, 2012.

23. Ustinova EE, Barrett CJ, Sun SY and Schultz HD: Oxidative stress impairs cardiac chemoreflexes in diabetic rats. Am J Physiol Heart Circ Physiol 279: H2176-H2187, 2000.

24. Gao X, Ohlander M, Jeppsson N, Björk L and Trajkovski V: Changes in antioxidant effects and their relationship to phytonutrients in fruits of sea buckthorn (Hippophae rhamnoides L.) during maturation. J Agric Food Chem 48: 1485-1490, 2000.

25. Rains JL and Jain SK: Oxidative stress, insulin signaling, and diabetes. Free Radic Biol Med 50: 567-575, 2011.

26. Itoh K, Wakabayashi N, Katoh Y, Ishii T, Igarashi K, Engel JD and Yamamoto M: Keap1 represses nuclear activation of antioxidant responsive elements by Nrf2 through binding to the amino-terminal Neh2 domain. Genes Dev 13: 76-86, 1999.

27. Ma Q, Kinneer K, Bi Y, Chan JY and Kan YW: Induction of murine $\mathrm{NAD}(\mathrm{P}) \mathrm{H}$ :quinone oxidoreductase by $2,3,7,8$-tetrachlorodibenzo-p-dioxin requires the CNC (cap ' $n$ ' collar) basic leucine zipper transcription factor Nrf2 (nuclear factor erythroid 2-related factor 2): cross-interaction between AhR (aryl hydrocarbon receptor) and Nrf2 signal transduction. Biochem J 377: 205-213, 2004.

28. He X, Chen MG, Lin GX and Ma Q: Arsenic induces NAD(P)H quinone oxidoreductase I by disrupting the $\mathrm{Nrf} 2 \cdot \mathrm{Keap} 1 \cdot \mathrm{Cul} 3$ complex and recruiting $\mathrm{Nrf} 2 \cdot \mathrm{Maf}$ to the antioxidant response element enhancer. J Biol Chem 281: 23620-23631, 2006.

29. Hardie DG: AMP-activated protein kinase: an energy sensor that regulates all aspects of cell function. Genes Dev 25: 1895-1908, 2011

30. Sriwijitkamol A, Ivy JL, Christ-Roberts C, DeFronzo RA, Mandarino LJ and Musi N: LKB1-AMPK signaling in muscle from obese insulin-resistant Zucker rats and effects of training. Am J Physiol Endocrinol Metab 290: E925-E932, 2006. 